\title{
Identification of Anticancer Lead Molecules against PRR11 Protein Target with Combination of Protein Modelling Through Threading Approach, Structure Based Chemical Screening of ZINC Database and Pharmacokinetic Properties
}

\author{
Sahukari Ravi, Punabaka Jyothi, Rajya Lakshmi, Bhasha Shanmugam, Ganjikunta Venkata Subbaiah, \\ Kesireddy Sathyavelu Reddy*
}

Department of Zoology, Division of Molecular Biology and Ethnopharmacology, Sri Venkateswara University, Tirupati, Andhra Pradesh, INDIA.

\begin{abstract}
PRR11 is a cell cycle regulator involved in pathogenesis of cancer. Therapeutically it has been identified as target to inhibit cancer growth. In this study, we employed in silico strategies for determining 3D structure of PRR11 and identification of lead molecules. Molecular modelling of PRR11 was carried out with the help of @TOME2, modeller 9.10 and ModLoop, further structure refinement conceded with SAVES server. ZINC database was virtually screened against reference marine natural compound and docking simulations were performed in AutoDock Vina. Pharmacokinetic properties of leads have been analysed in DataWarrior. Collectively, the results revealed that the 3D structure of target was successfully build with refinement of 30 loops, the reliable structure showed that $83.87 \%$ amino acid residues present in allowed regions of Ramachandran plot, Errat overall quality factor was 83.807 , passed in Prove and more than $65 \%$ amino acids have scored $>=0.2$ in the 3D/1D plot of PRR11. Based on small molecule screening of ZINC database against PRR11 with resulted pharmacokinetic descriptors, 5 lead molecules (28863059, 79642438, 27766155, 27855322 and 28365298) have predicted as PRR1 1 inhibitors, which are very prominent in binding energy values, ADME and adverse effects (MTIR) calculations than reference marine natural compound. ZINC database has been given a choice to purchase lead molecules for further evaluations in in vitro/in vivo studies; it could be an opportunity to further prove of this in silico predictions.
\end{abstract}

Key words: PRR11, ZINC Database, Threading, Docking, Pharmacokinetics.

\section{INTRODUCTION}

Cancer is a complex disease, characteristically it initiates as benign tumor at specific organ of the body, but its prevalence taken to the other body parts by malignance mechanism. Genes are deviated into two major types in the cancer phenotype; they are proto oncogenes and tumor suppressor genes; mutations in these genes are consequences the growth of cancer. $^{1,2}$ Cancer is commonly well thought of a cell cycle disease. Cell cycle is a sequentially ordered and highly regulated mechanism for the division of a cell, which is essential for transmission of genetic material from one generation to the next. ${ }^{3}$ Huge number of cell cycle regulators influence the cell division, if such regulators are altered/ over expressed, this will lead to unrestrained cell proliferation.

PRR11 is a cell cycle regulator, its expression vary in cell cycle stages. ${ }^{4}$ PRR11 function has been recently identified in various cancer
Submission Date: 01-09-2017; Revision Date: 05-12-2017; Accepted Date: 19-12-17

DOI: 10.5530/ijper.52.3.44 Correspondence: Dr. Kesireddy Sathyavelu Reddy,

Professor, Division of Molecular Biology and Ethnopharmacology, Department of Zoology, Sri Venkateswara University, Tirupati - 517 502, INDIA.

Phone: $+91-877-2248892$ (Off)

E-mail: ksreddy2008@ hotmail.com

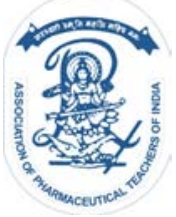

www.ijper.org 
types. It's over expression has been observed at both $\mathrm{m}$-RNA and protein levels in lung cancer tissue. It has been silenced by RNAi, then it resulted $\mathrm{S}$ phase arrest, suppressed cellular proliferation, cell migration, invasion and colony forming ability in lung cancer cells whereas, growth retardation and $\mathrm{S}$ phase arrest was observed in HeLa cells; also inhibited tumorogenic property in nude mice. Micro array analysis of lung cancer tissue was shown that its knock down dysregulates the various genes involved in metastasis, tumorigenesis and cell cycle. ${ }^{5}$ Zhang et al., (2012) ${ }^{6}$ reported that PRR11 gene over expression associated with poor survival rate of pancreatic cancer patients.

Human genome project has been taken a lot of investment for the identification of complete genome of human. At present, the ratio of protein sequences are available in UniProt database and the protein crystal/NMR structures deposited in PDB (protein data bank) is distant, at least $10 \%$ of sequences are not determined into structures but most of them are well-known as therapeutic targets of diseases. Meanwhile their suppression by RNAi and other technologies curing the diseases were experimentally proved. Hence, target protein atomic and molecular structure through X-ray crystallography and NMR, further identification of drugs against such targets is urgent necessary. ${ }^{7,8}$ Determination of protein structures through experimental methods are tended to very slow and not succeed for all proteins especially for membrane proteins. ${ }^{9}$ Bioinformatics is a new discipline emerged from engage of biology and computer science, it is a site of address for many complicated questions which are not answered by experimental methods. For example, now a day structural biologists determining the 3D structure of target proteins through molecular modelling approaches computationally for many sequences, with the help of homology sequence crystal structures present in PDB. ${ }^{10}$ Also, pharmacologists identifying the lead molecules against modelled structures through structure based virtual screening of small molecule libraries. ${ }^{11}$

This study has been planned mainly to determine PRR11 protein 3D structure (macro molecule), a therapeutic target of cancer with threading application of molecular modelling. Further, validations of modelled structure were carried out through web servers. Next to model, identification of ligand binding sites in structure and screening of ZINC database against them through structure based virtual screening approach in AutoDock Vina software. Additionally, the pharmacokinetic properties of leads (micro molecules) and their adverse effects were predicted with computational tools.

\section{MATERIALS AND METHODS}

\section{Molecular threading and modelling of macromolecule}

The PRR11 protein was identified as target in cancer but no crystallographic structure is available in the PDB (Protein data bank) yet. Based on crystal structure of template (similar protein) homology with target sequence, a computational crystallography method of modeller for proteins was developed by Sali and Blundell, (1993). ${ }^{12}$ The protein sequence retrieved from UniProt database and searched for template crystal structure in@TOME2, a threading server. ${ }^{13}$ Before modelling, the evolutional relationship of two protein (PRR11- Template) sequences was confirmed by alignment event using ClustalW tool. The template structure (PDB: 1tvk "the binding mode of epothilone A on alpha, beta-tubulin"), which is identified by threading server.), target sequence and necessary scripts for modelling were run on the modeller $9.10 \mathrm{v}$ platform. Total 200 simulations for protein modelling were made and fine five 3D model structures of target protein were selected based on Discrete Optimized Protein Energy (DOPE) scores.

\section{Target protein refinement}

The final refinement of selected 5 target protein structures were evaluated in SAVES server, a domain with collective protein evaluation parameters. ${ }^{14}$ In broad spectrum of protein biology, protein models are being subjected to refinement for their bond orders, missing hydrogen atoms, topologies and other missing groups. ${ }^{15}$ The stereo chemical quality in modelled protein was explored with Ramachandran plot and Whatcheck parameters; the error functions of residues and non bonded interactions between atoms of structure was observed using Errat; and compatibility of every amino acid of PRR11sequence with final 3D structure was calculated using Verify3D. Based on all the values, one refined 3D structure was selected for further loop refinement with ModLoop server, a modeller associated web server. ${ }^{16}$ Homology sequences are not completely conserved during evolution, so possibility of insertions / deletions often occur called loops. These are variable regions determine the functional specificity by contributing activity and binding sites of protein structure. Loop modelling accuracy determines the usefulness of homology model in docking studies. ${ }^{17}$

\section{Ligand binding site prediction}

Generally, structure based virtual screening initiated with detection of ligand binding sites in proteins; 
these sites are pocket like arrangements with molecular adherence surfaces, hydrogen bond donors, hydrogen bond acceptors and hydrophobic features. ${ }^{18}$ The proteins/ enzymes (macro molecules) with co-crystal structures make clear its drug/lead (micro molecules) binding sites; those reflect their functional parts along with key amino acids, which are interacted with micro molecules. In case of uncrystallized macro structures, the micro molecules binding sites needs to be predicted. Today, ligand binding sites of protein being achieved through web servers. ${ }^{19}$ These are predicting the sites based on amino acid composition and folds. CASTp server is the one of best binding site predictor ${ }^{20}$ hence, it has been used in this study to find out ligand binding sites of PRR11.

\section{Micro molecules selection and structure based virtual screening}

In this approach, we selected anticancer marine source natural products and their derivatives as reference compounds for initial docking. At present, these compounds are at the level of preclinical and clinical trials against cancer. The docking simulations were carried out in the AutoDock Vina wizard of PyRx software. ${ }^{21}$ Initial docking resulted best fitted compound was used for further virtual screening of ZINC database, a place of library for micro molecules. Obtained analogue data set of initial best compound was redocked into the identified binding sites of macromolecule. The docking simulations executed in AutoDock Vina based on genetic algorithm; it simulates each compound for eight iterations (stereo chemical rotation of bonds present in the chemical compound) in the binding site and calculates the binding energy in the form of $\mathrm{kcal} /$ mole.

\section{Pharmacokinetic calculations and molecular visualization}

Based on structural information available for the present drugs, computational methods have been developed, those are predict the drug pharmacokinetic properties like ADME and adverse effects such as mutagenicity, tumorogencity, irritation and reproductive effect (MTIR). DataWarrior 4.4.4 version, ${ }^{22}$ a product of Osiris server, is an excellent drug property filter, which has been used to calculate the properties of lead molecules. Structure based virtual screening resulted lead compounds interaction with amino acids of PRR11 need to be visualized for the beauty view and easy understanding. Finally, this mark has been succeeded with the use of PyMOL tool, freely accessible and user friendly molecular visualizer.

\section{RESULTS AND DISCUSSION}

\section{Molecular threading and modelling of PRR11}

The cells equipped with huge number of proteins for their native function and homeostasis. The protein sequences deposited in UniProt is above 20 million but their crystal structures deposited in PDB is only 90,000, this give us a clear idea that the challenge in determining crystal structures of proteins is arduous. ${ }^{8}$ The protein targeted chemo therapy being played a vital role in the treatment of diseases. Due to insufficient crystal structures of target proteins, the bioinformatics tools taken a step to build 3D models based on homology of template crystal structures of others. In this study, we found moderate relation score upon sequence alignment of template (PDB: 1tvk) and PRR11 sequences. The alignment of template and PRR11 is shown in Figure 1. Further, we build a 3D model of PRR11 through threading approach with modeller assistance. Among 200 modelled structures from modeller, the best DOPE value structures were selected; their scores are -27226.66797, -27090.89648, -27048.83984, -27017.51563 , and -27016.26172 .

\section{Structural evaluation of protein}

Initially, all 3D structures evaluated through Ramachandran plot, Errat, Whatchek and Verify3D. Next to that, data obtained from above parameters were analysed and best model (DOPE: -27090.89648) was selected. Final refinement of this model was increased by identification of unfolded amino acids in superimposed template with target using PyMOL and its modelling was done through ModLoop server. Total 30 loops (Table 1) were refined to make the PRR11 structure valid and final validation reports except Ramachandran plot were represented in Figure 2 whereas Ramachandran plot and validated final macro molecule 3D structure portray in Figure 3; obtained validation values are as follows, $82.7 \%$ amino acids present in allowed regions of Ramachandran plot; the verify3D value resulted as $66.65 \%$ and Errat was 83.807 , all these parameters confirmed the structural reliability and strengthen its utility for further studies.

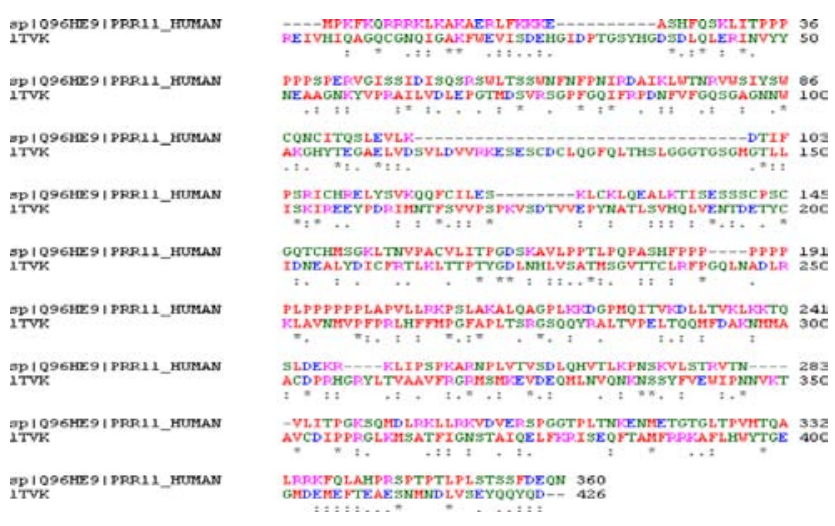

Figure 1: Sequence alignment of target (PRR11) protein and template in clustal W 
Table 1: Unfolded regions identified in PyMOL when template-3D model of PRR11 alignment, which are simulated to fold using ModLoop server.

Loop Region and sequence

${ }_{2} \mathrm{PKF}_{4} ;{ }_{17} \mathrm{RLFKKKEA}_{24} ;{ }_{38} \mathrm{PPS}_{40} ;{ }_{49} \mathrm{IDIS}_{52} ;{ }_{56} \mathrm{SW}_{57} ;{ }_{64} \mathrm{FNF}_{66} ;{ }_{70} \mathrm{RDAIKLW}_{76} ;{ }_{78} \mathrm{NRV}_{80} ;{ }_{89} \mathrm{NCl}_{91} ;{ }_{98} \mathrm{LKDTI}_{102} ;{ }_{108} \mathrm{CHRE}_{111} ;{ }_{119} \mathrm{FCIL}_{122} ;{ }_{127} \mathrm{CKLQ}_{30} ;$ ${ }_{148} \mathrm{TCH}_{150} ;{ }_{157} \mathrm{NVPA}_{160} ;{ }_{170} \mathrm{KAVL}_{173} ;{ }_{204} \mathrm{LLRK}_{207} ;{ }_{209} \mathrm{SLAKALQA}{ }_{216} ;{ }_{221} \mathrm{KDG}_{223} ;{ }_{228} \mathrm{TVKD}_{231} ;{ }_{239} \mathrm{KTQ}_{241} ;{ }_{265} \mathrm{LQHV}_{268} ;{ }_{270} \mathrm{LKPN}_{273} ;{ }_{279} \mathrm{TR}_{280} ;{ }_{283} \mathrm{NV}_{284} ;$ ${ }_{287} \mathrm{TP}_{288} ; 290 \mathrm{KSQ}_{292} ;{ }_{299} \mathrm{LRKVDVERSPGGT}_{311} ;{ }_{329} \mathrm{MT}_{330}$ and ${ }_{337} \mathrm{FQ}_{338}$.
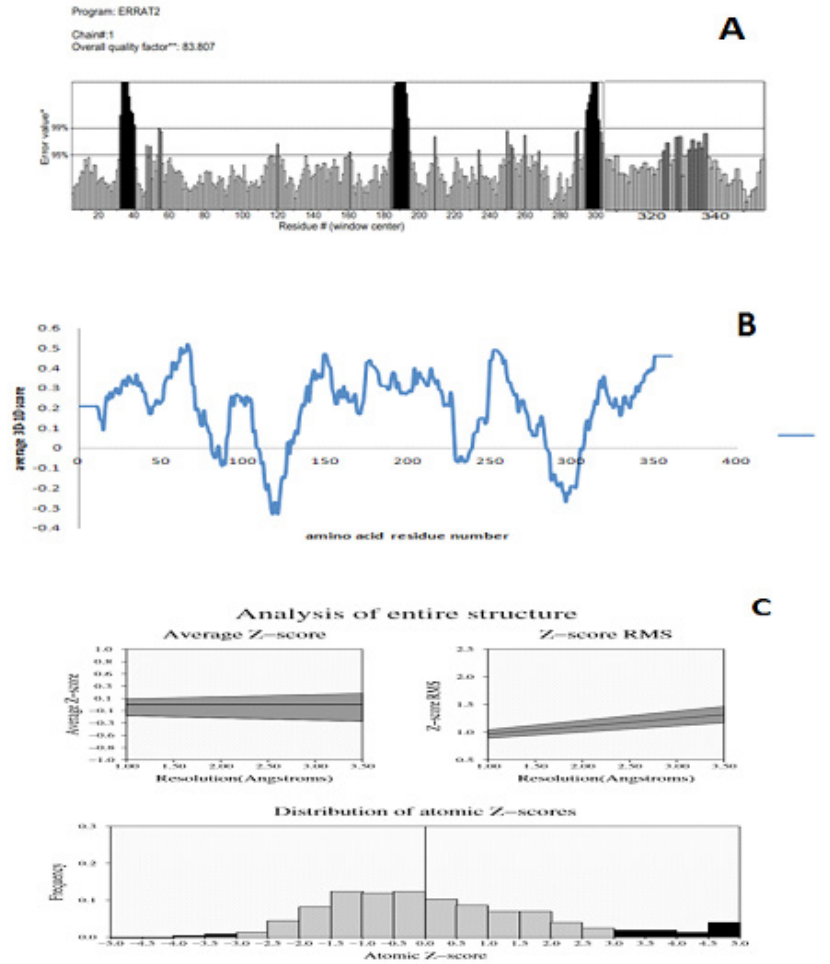

Figure 2: PRR11 protein evaluation using SAVES server: A) the non-bonded interactions between atoms types were computed with ERRAT program which shows overall quality factor. B) Analysis of the compatibility of an atomic model (3D) with its own each amino acid residvbbue assigned a structural class based on its local and environment in Verify 3D. C) PROVE calculates the volume of atoms in macro molecule and treats atoms like hard spheres, finally calculates statistical Z-scores deviation.

\section{Docking and screening of ZINC database}

After successful improvement of 3D model of PRR11 protein, initial docking was carried out with marine anticancer co-ordinates as micro molecular agents. AutoDock Vina compatible files uploaded include ligands as structure data files and macro molecule as protein data bank file. Initial docking explored HTI-286 (Hemiasterlin) as a reference compound with binding energy $-9.5 \mathrm{kcal} / \mathrm{mole}$ in the binding site 1 and -9.1 $\mathrm{kcal} /$ mole in the binding site 2 (Figure 4). The auto grid coordinates (X: 51.2911 Y: 58.1411 Z: 57.6582) accepted the natural compounds free iterations into the binding sites. Further, the ZINC database screening
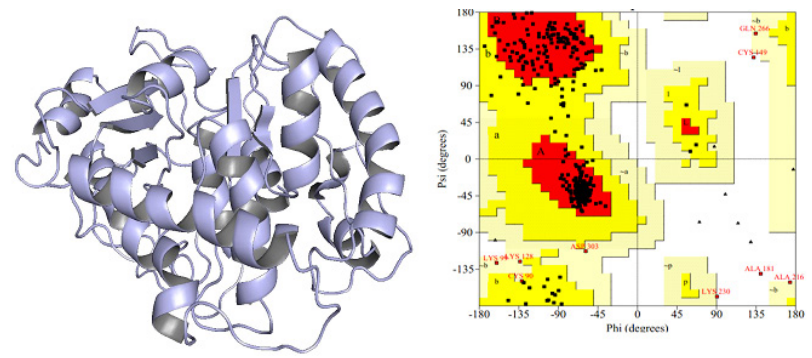

Figure 3: Refined model of PRR11 (Left) and the refinement report depicted using Ramachandran plot (Right).

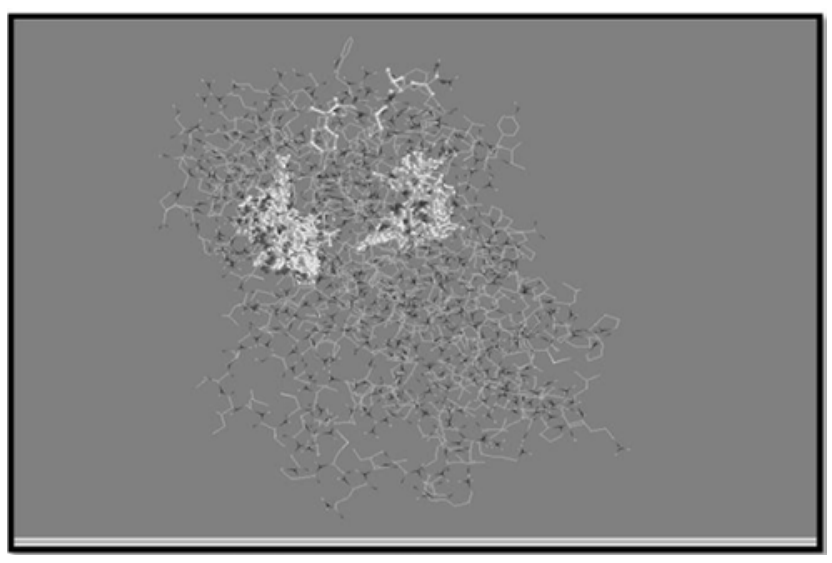

Figure 4: Docking geometry of ZINC small chemical molecules in binding sites of PRR11.

for the Hemiasterlin relevant structural features comes out about 3,440 homology hits. Those were redocked in same constructed auto grid in the protein. Though, more number of molecules with best ranking than reference compound was explored and after filtration of pharmacokinetic properties, we selected five lead molecules which are discussed below.

\section{Drug property filtration}

Any chemical constituent with potential activity should follow some rules and regulations for further formulation and oral prescriptions. Lipinski et al., $(1997)^{23}$ predicted the rule of five for FDA approved oral drugs, they finalized that orally accepted drugs need 
Table 2: Drug properties like Lipinski rule of five was observed in Molinspiration and drug adverse effects studied in Osiris server.

\begin{tabular}{|c|c|c|c|c|c|c|c|c|c|c|c|c|c|}
\hline S.No & Zinc id & MW & cLogp & HD & HA & TPSA & nRB & SL & D S & MuG & TuG & IrT & RE \\
\hline 1. & 28863059 & 692.818 & 0.43 & 4 & 15 & 185.764 & 12 & -1.3 & 0.55 & NO & NO & NO & NO \\
\hline 2 & 79642438 & 444.599 & 4.29 & 4 & 5 & 77.049 & 13 & -5.22 & 0.3 & NO & Partially & NO & NO \\
\hline 3 & 27766155 & 586.713 & 1.69 & 8 & 10 & 169.469 & 11 & -4.42 & 0.51 & NO & NO & NO & NO \\
\hline 4 & 27855322 & 586.713 & 1.69 & 8 & 10 & 169.469 & 6 & -4.42 & 0.51 & NO & NO & NO & NO \\
\hline 5 & 28365298 & 565.755 & 4.28 & 3 & 8 & 115.374 & 6 & -4.55 & 0.43 & NO & NO & NO & NO \\
\hline 6 & $\begin{array}{c}\text { HTI-286 } \\
\text { Hemiasterlin } \\
\text { Derivative }\end{array}$ & 473.655 & 2.28 & 3 & 7 & 98.74 & 11 & -3.515 & 0.35 & NO & NO & NO & NO \\
\hline
\end{tabular}

MW:Molecular Weight. HD:Hydrogen Bond Donars. HA: Hydrogen Bond Acceptor. TPSA:Topological polar surface area, Nrb: No of Rotatable Bonds. SL: Solubility. DS: Drug Score. Mug: Mutagenicity. Tug:Tumarogenicity. Irt: Irritant. RE: Reproductive Effect.

to have molecular weight $(\mathrm{MW})<500$, hydrogen bond donors $<500$, hydrogen bond acceptor $<10$ and octanolwater partition coefficient $(\log \mathrm{P})<5$. Lipinski rule of five is a drug property filter, describes ADME properties. But, Young and Mark $(2011)^{24}$ found that few non oral drugs approved by FDA have violated the rule of five, especially ophthalmic drugs in MW, hydrogen bond acceptors over the threshold number. Many drugs available in the market for the treatment of diseases are showing adverse effects, those are mainly MTIR. Both filters have been carried out for the top five ligands using Data Warrior. All the compounds have been qualified most of the predicted pharmacokinetic properties which are represented in Table 2. Compounds such as ZINC 28863059, 79642438, 27766155, 27855322 and 28365298 were selected for molecular visualizations. The selected compounds have qualified ADME except molecular weight digit (MW more than 500 except ZINC 79642438). It is clearly indicates that the lead ZINC 79642438 as oral and other all leads are confidence to prescribe as non oral drugs. The ZINC 79642438 lead has showing partial tumorogencity whereas rest of leads are not having adverse effects.

\section{Macro-micro molecules interactions}

The lead molecules with least binding energy in binding site and presence of hydrogen bond donor/acceptor descriptors count are play crucial role in molecular interactions. In this work, molecular interactions have been visualized and identified in PyMOL tool, where the protein was exposed as cartoon and the leads are in the form of sticks. Based on binding energy values, we selected top ten leads but after pharmacokinetic property prediction, five of them were omitted. The leads 28863059, 79642438, 27766155, 27855322, 28365298 with common prefix ZINC have been finally selected (Table 3). The best ranking given to the leads based on binding energy values in binding sites of PRR11, their molecular interactions are summarized below. The ZINC 28863059 donated two hydrogen atoms $\left(\mathrm{H}_{34}-\mathrm{N}_{17}\right.$ and $\mathrm{H}_{47}-\mathrm{N}_{45}$ ) to the receivers of $\mathrm{SER}_{40}$ and $\mathrm{LYS}_{170}$ amino acids; whereas $\mathrm{MET}_{1}$ nitrogen atom (CA-N) acted as a hydrogen bond donor to the carbonyl oxygen of lead molecule in respect of hydrogen bond interactions. Two hydrogen bond interactions have been formed by the 79642438 through one hydrogen bond acceptor and one donor to the carbonyl group oxygen of $\mathrm{LYS}_{99}$ and Nitrogen atom of TRP ${ }_{62}$ respectively. Among 8 hydrogen bond donors and 10 hydrogen bond acceptors of the ZINC 27766155, two donors $\left(\mathrm{H}_{17}-\mathrm{N}_{16}, \mathrm{H}_{18}-\mathrm{N}_{16}\right)$ interacted with $\mathrm{GLU}_{96}, \mathrm{GLN}_{117}$ and one acceptor $\left(\mathrm{O}_{45}-\mathrm{C}_{42}\right)$ bonded to $\mathrm{GLN}_{93}$ of PRR11 binding site amino acids. Ligand binding site amino acids such as $\mathrm{TRP}_{86}, \mathrm{GLN}_{93}$ and $\mathrm{GLU}_{111}$ have formed bonds with hydrogen bond donors such as $\mathrm{H} 49-\mathrm{N}_{47}, \mathrm{H} 17-\mathrm{N}_{16}$ and $\mathrm{H} 46-\mathrm{O}_{45}$ respectively of ZINC 27855322. The lead ZINC 28365298 was formed three hydrogen bond interactions through its hydrogen bond acceptors $\left(\mathrm{O}_{28}-\mathrm{C}_{27}, \mathrm{O}_{13}{ }^{-} \mathrm{C}_{12}\right.$ and $\left.\mathrm{O}_{14}{ }^{-} \mathrm{C}_{12}\right)$ to the common hydrogen bond donor $(\mathrm{CN})$ of $\mathrm{LYS}_{30}$, $\mathrm{CYS}_{90}$ and $\mathrm{GLN}_{93}$ respectively. The molecular visual/ value of docking results such as binding energy, bonding interactions, bond angle and bond lengths are illustrated in Table 3.

\section{CONCLUSION}

Virtually screened ZINC database against PRR11 therapeutic target explored the lead molecules, are include ZINC 28863059, 79642438, 27766155, 27855322 and 28365298. These leads have been showed good binding energies with qualified drug properties and without adverse effects than reference compound. $\mathrm{MET}_{1}$, $\mathrm{SER}_{40}, \mathrm{LYS}_{170}, \mathrm{TRP}_{62}, \mathrm{LYS}_{99}, \mathrm{GLN}_{93}, \mathrm{GLU}_{96}, \mathrm{GLN}_{117}$, $\mathrm{TRP}_{80}, \mathrm{GLU}_{11}, \mathrm{LYS}_{30}$ and $\mathrm{CYS}_{90}$ are key amino acids of PRR11, involved in hydrogen bond interactions with leads. Lead molecules are available in ZINC database 


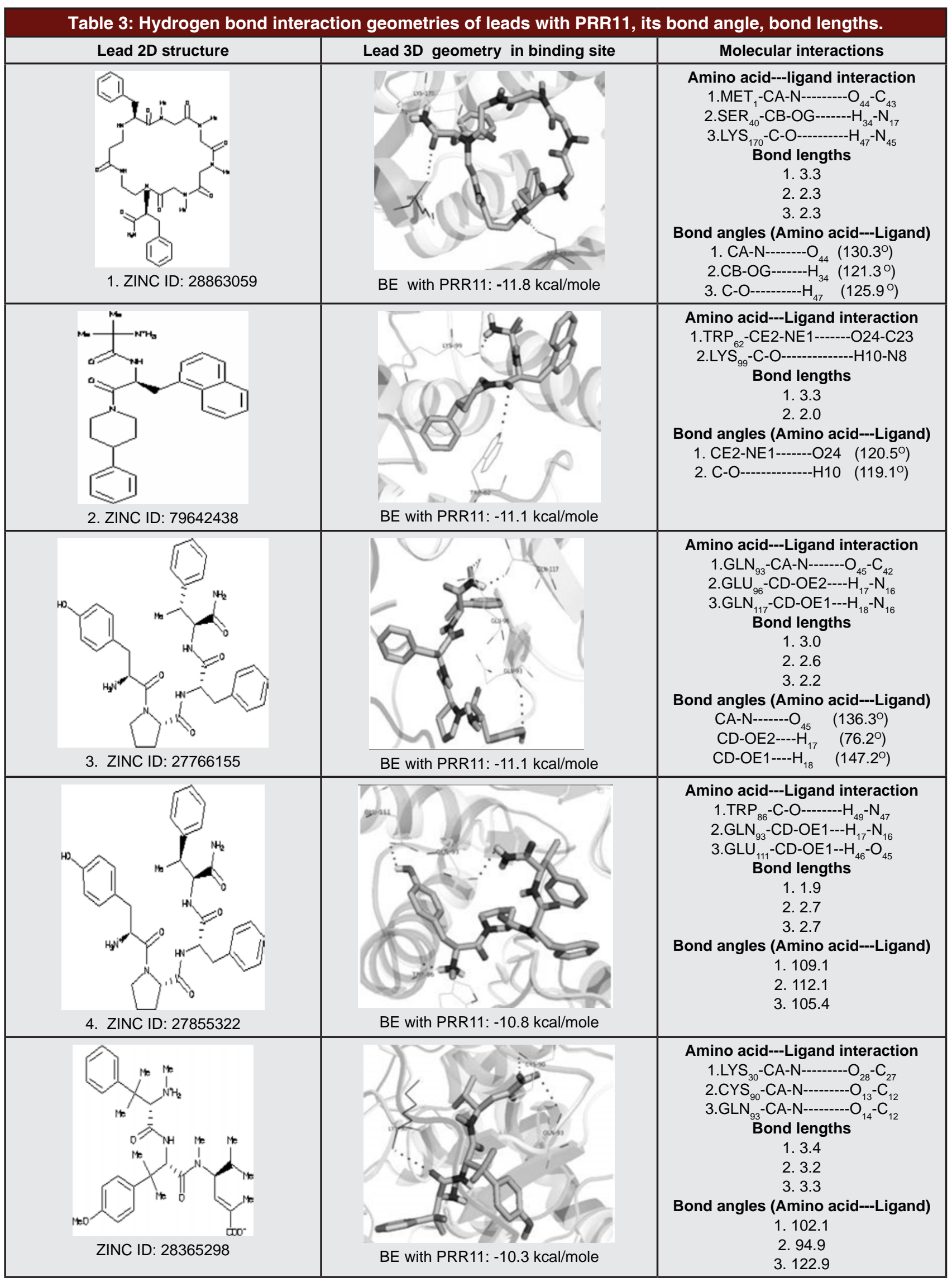

Continued... 


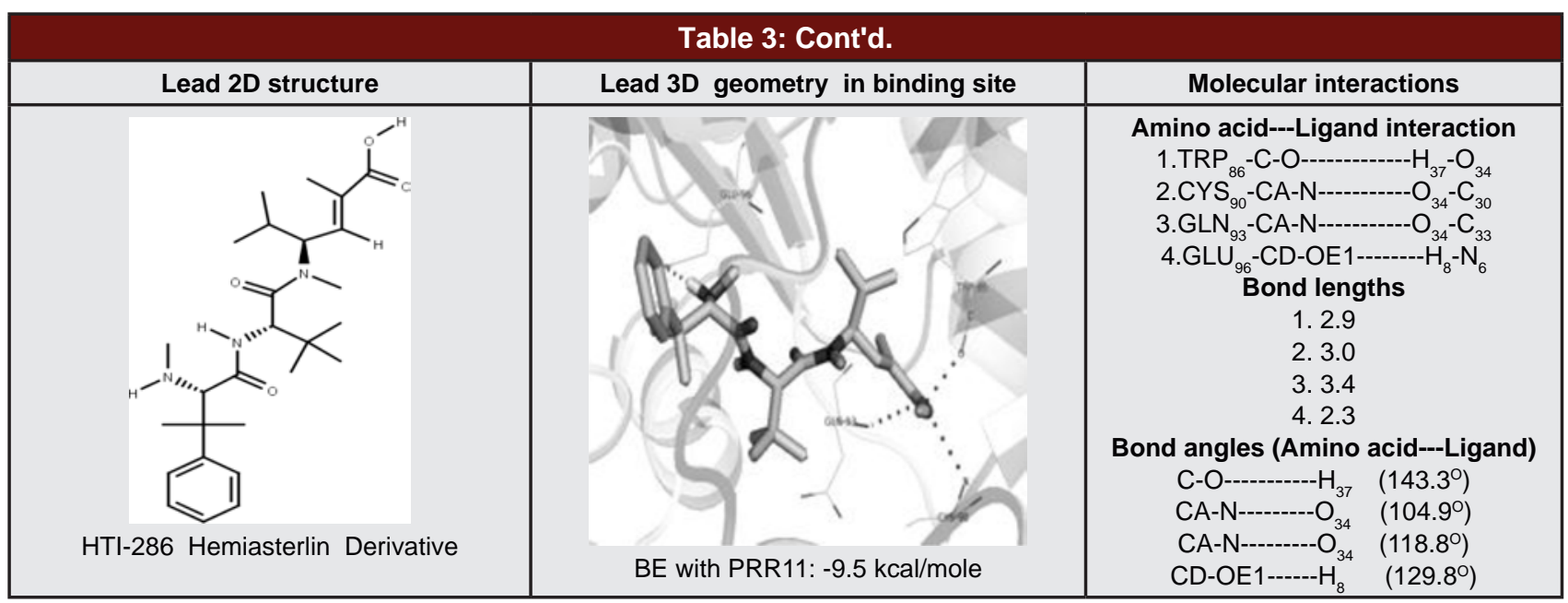

\section{BE: Binding energy}

for purchase; hence it could give a chance to prove this in silico study results in in vitro and in vivo experiments. If it could, the significance of interacted amino acids of PRR11 with lead molecules also possible then functional moieties of protein would explore.

\section{ACKNOWLEDGEMENT}

All authors express sincere gratitude to the University Grants Commission New Delhi, India for the financial assistance, especially to the first and second authors (SR and PJ) in the form of RGNF (No: F117.1/201213/R GNF-2012-13-SC-AND 34335; and No: F1-17.1/2015-16/RGNF-2015-17-SC-AND-17449 respectively) and to the corresponding author (KSR) through Major Research Project (No: 43-586/2014 (SR), Dated 30-10-2015).

\section{CONFLICT OF INTEREST}

All authors are disclose that there is no conflict of interest

\section{ABBREVIATIONS}

PRR11: Proline rich protein 11; MTIR: Mutagenicity, tumarogenicity, irritant and reproductive effect; ADME:"Absorption, distribution, metabolism, and excretion; PDB: Protein data bank; MW: Molecular weight; HD: Hydrogen bond donor; HA: Hydrogen bond acceptors; TPSA: Topological polar surface area; Nrb: No of Rotatable Bonds; SL: Solubility; DS: Drug Score.

\section{REFERENCES}

1. Futreal PA, Coin L, Marshall M, Down T, Hubbard T, Wooster R, et al. A census of human cancer genes. Nat Rev Cancer. 2004;4(3):177-83. doi: 10.1038/nrc1299. PMid: 14993899.
2. Hanahan D, Weinberg RA. Hallmarks of cancer: the next generation Cell. 2011;144(5):646-74. doi.org/10.1016/j.cell.2011.02.013. PMid:21376230.

3. Israels ED, Israels LG. The cell cycle. Oncologist. 2000;5:510-3. doi: 10.1634/theoncologist.5-6-510.

4. Larance M, Ahmad Y, Kirkwood KJ, Ly T, Lamond Al. Global Subcellular Characterization of Protein Degradation Using Quantitative Proteomics. Mol Cell Proteomics. 2013;12(3):638-50. doi: 10.1074/mcp.M112.024547. PMid: 23242552.

5. Ji Y, Xie M, Lan H, Zhang $\mathrm{Y}$, Long $\mathrm{Y}$, Weng $\mathrm{H}$, et al. PRR11 is a novel gene implicated in cell cycle progression and lung cancer. Int J Biochem Cell Biol. 2013;45(3):645-56. doi: 10.1016/j.biocel.2012.12.002. PMid: 23246489.

6. Zhang G, Schetter A, He P, Funamizu N, Gaedcke J, Ghadimi BM, et al. DPEP1 Inhibits Tumor Cell Invasiveness, Enhances Chemosensitivity and Predicts Clinical Outcome in Pancreatic Ductal Adenocarcinoma. PLoS One. 2012;7(2):e31507. doi:10.1371/journal.pone.0031507. PMid: 22363658.

7. Tramontano A. Minireview: The role of molecular modelling in biomedical research. FEBS Lett. 2006;580(12):2928-34. doi: 10.1016/j. febslet.2006.04.011. PMid: 16647064

8. Russo KI, Merlino A, Vergara A, Sica F. An Overview of Biological Macromolecule Crystallization. Int J Mol Sci. 2013;14(6):11643-91. doi: 10.3390/ijms140611643. PMid:23727935.

9. Floudas CA, Fung HK, McAllister SR, Mönnigmann M, Rajgaria R. Advances in protein structure prediction and de novo protein design: A review. Chem Eng Sci. 2006;61(3):966-88. doi.org/10.1016/j.ces.2005.04.009.

10. Vyas VK, Ukawala RD, Ghate M, Chintha C. Homology Modeling a Fast Tool for Drug Discovery: Current Perspectives. Indian J Pharm Sci. 2012;74(1):117. doi: 10.4103/0250-474X.102537.

11. Lionta E, Spyrou G, Vassilatis DK, Cournia Z. Structure-Based Virtual Screening for Drug Discovery: Principles, Applications and Recent Advances. Curr Top Med Chem. 2014;14(16):1923-38. doi: 10.2174/15680266146661 40929124445. PMid: 25262799.

12. Sali A, Blundell TL. Comparative protein modelling by satisfaction of spatial restraints. J Mol Biol. 1993;234(3):779-815. doi.org/10.1006/jmbi.1993.1626. PMid: 8254673.

13. Pons JL, Labesse G. @TOME-2: a new pipeline for comparative modeling of protein-ligand complexes. Nucleic Acids Res. 2009;37(2):485-91. doi: 10.1093/nar/gkp368. PMid: 19443448

14. Kalirajan R, Sankar S, Jubie S, Gowramma B. Molecular Docking studies and in-silico ADMET Screening of Some novel Oxazine substituted 9-Anilinoacridines as Topoisomerase II Inhibitors. Ind J Pharm Edu Res. 2017;51(1):110-5. doi: 10.5530/ijper.51.1.15.

15. https://services.mbi.ucla.edu/SAVES/

16. Fiser A, Sali A. ModLoop: automated modeling of loops in protein structures. Bioinformatics. 2003;19(18):2500-1. PMid:14668246 
17. Lee J, Lee D, Park H, Coutsias EA, Seok C. Proteinloop modeling by using fragment assembly and analytical loop closure. Proteins. 2010;78(16):3428-36. doi: 10.1002/prot.22849. PMid: 20872556.

18. Munichandrababu T, Bhaskar BV, Ravi S, Bhuvaneswar C, Rajendra W. Structure based virtual screening of non-steroidal antiinflammatory drugs (NSAIDs) against RNA-binding motif 6 (RBM6) involved in human lung cancer. Med Chem Res. 2013;22(6):2828-39. doi: 10.1007/s00044-012-0276-7

19. Kinoshita $\mathrm{K}$, Nakamura $\mathrm{H}$. Identification of the ligand binding sites on the molecular surface of proteins. Protein Sci. 2005;14(3):711-8. doi: 10.1110/ ps.041080105. PMid:15689509.

20. Binkowski TA, Naghibzadeh S, Liang J. CASTp: Computed Atlas of Surface Topography of proteins. Nucleic Acids Res. 2003;31(13):3352-5. PMid:12824325.
21. Trott O, Olson AJ. AutoDock Vina: improving the speed and accuracy of docking with a new scoring function, efficient optimization and multithreading. J. Comput. Chem. 2010;31(2):455-61. doi: 10.1002/jcc.21334. PMCid: PMC3041641

22. Sander T, Freyss J, Von KM, Rufener C. DataWarrior: an open-source program for chemistry aware data visualization and analysis. J Chem Inf Model. 2015;55(2):460-73. doi: 10.1021/ci500588j. PMid: 25558886.

23. Lipinski CA, Lombardo F, Dominy BW, Feeney PJ. Experimental and computational approaches to estimate solubility and permeability in drug discovery and development settings. Adv Drug Deliv Rev. 2001;46 (1-3):3-26. PMid:11259830.

24. Choy YB, Prausnitz MR. The Rule of Five for Non-Oral Routes of Drug Delivery: Ophthalmic, Inhalation and Transdermal. Pharm Res 2011;28(5):943-8. doi 10.1007/s11095-010-0292-6.

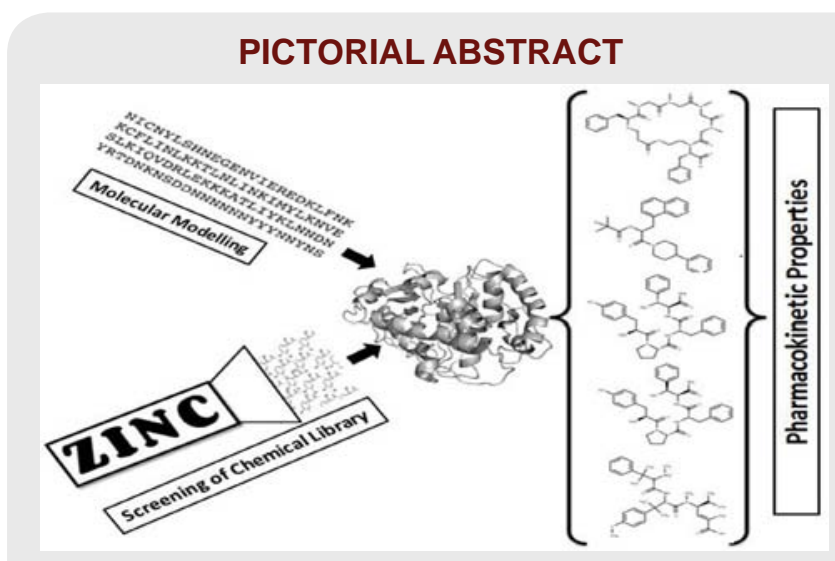

\section{SUMMARY}

- Identification of lead molecules against therapeutic targets like PRR11 of cancer is an essential area of today's research.

- In this study, application of protein modelling tools for homology modelling and threading approaches determined the 3D structure of PRR11.

- Screening of small chemical molecule library (ZINC database) against ligand binding sites of PRR11 explored the five best lead molecules (28863059, 79642438, 27766155, 27855322 and 28365298).

- Computational prediction of ADME and adverse characters (MTIR) confirmed that all identified leads are safe for further invitro and invivo experimental evaluations.

Cite this article: Ravi S, Jyothi P, Lakshmi R, Shanmugam B, Subbaiah GV, Reddy KS. Identification of Anticancer Lead Molecules against PRR11 Protein Target with Combination of Protein Modelling Through Threading Approach, Structure Based Chemical Screening of ZINC Database and Pharmacokinetic Properties. Indian J of Pharmaceutical Education and Research. 2018;52(3):381-8. 